\title{
Exploratory study on illegal pharmacologic agents in mixed martial arts performance
}

\section{Estudo exploratório sobre agentes farmacológicos ilegais no desempenho em artes marciais mistas}

\author{
Ciro José Brito \\ Rayssa Lodi Mozer \\ Erasmo Montes Assis de Bem \\ Pedro Henrique Berbert de Carvalho \\ Andréia Cristiane Carrenho Queiroz ${ }^{1}$ \\ Fábio dal Bello² \\ Lindsei Brabec Mota Barreto ${ }^{3}$ \\ Bianca Miarka ${ }^{1}$
}

Abstract - Mixed Martial Arts (MMA) is a combat sport that requires maximum physical effort during competitions. In this context, some athletes can use illicit substances in order to improve their performance. By means of paired analysis, the present study compared the motor actions of athletes who had failed an anti-doping test versus their performance in combat against a winner or loser without doping presence. For this, 267 rounds (male and female) were analyzed in professional matches. The rounds were paired by athletes in the conditions: doping, winning and losing. Motor actions were analyzed through a specific and previously-validated protocol. Of the substances detected, anabolic androgenic steroids represented 55\% ( $\mathrm{p} \leq 0.001)$. Doped athletes had lower pause time $(83.4 \pm 68.3$ vs. $131.7 \pm 95.2, p \leq 0.001)$ and longer time at high-intensity $(85.2 \pm 86.6$ vs. $51.2 \pm 73.3, p=0.002)$ compared to the losing condition. Regarding the technical-tactical analysis in standing combat, winning presented a higher mean compared to doping in all variables except for Knockdowns ( $\mathrm{p}=0.08)$, single body strikes landed ( $\mathrm{p}=0.15)$, single leg strikes landed $(p=0.25)$ and single strike attempts $(p=0.4)$. In conclusion, athletes who tested positive presented higher performance in the physical variables (effort and pause time) in comparison to the losing condition; however, doping did not reflect in better technical-tactical performance.

Key words: Athletic performance; Doping in sports; Martial arts; Time motion studies.

Resumo - O Mixed Martial Arts (MMA) é um esporte de combate que exige a máxima capacidade física durante as competiçôes, neste contexto, alguns atletas podem administrar substâncias ilícitas, com fins de ampliar artificialmente sua performance. Por meio de análise pareada, o presente estudo comparou as açôes motoras de lutadores flagrados no teste de doping frente ao desempenho em combates em que o mesmo saiu vencedor ou foi derrotado sem a presença de doping. Para tal, foram analisados 267 rounds em combates profissionais (masculino e feminino). Os rounds foram pareados por lutadores nas condiçôes: doping, vencedor e perdedor. As açôes motoras foram analisadas através de protocolo específico previamente validado. Das substâncias detectadas, os esteroides anabólicos androgênicos representaram (55\%; $p \leq 0,001)$. Lutadores dopados apresentaram menor tempo de pausa $(83,4 \pm 68,3$ vs. 131,7 $\pm 95,2 ; p \leq 0,001)$ e maior tempo em alta intensidade $(85,2 \pm 86,6$ vs. 51,2 $\pm 73,3 ; p=0,002)$ frente a condição Derrota. Quanto a análise técnico-tática em combate em pé, a condição Vitória apresentou maior média frente ao doping em todas as variáveis exceto Knockdowns $(p=0,08)$, golpe simples acertado no corpo $(p=0,15)$, e na perna $(p=0,25)$ e tentativa de golpe simples $(p=0,4)$. Em conclusão, lutadores que testaram positivo apresentaram maior desempenho nas variáveis físicas (tempo de esforço e tempo de pausa) frente a condição derrota; no entanto, a condição de doping não refletiu melhor desempenho técnico.

Palavras-chave: Artes marciais; Dopping nos esportes; Desempenho atlético; Estudos de tempo e movimento.
1 Federal University of Juiz de Fora. Campus Governador Valadares, MG. Brazil.

2 Universidad Santo Tomás. Physical Activity and Sports Science Master Program. Santiago. Chile.

3 Secretariat of Education of the State of Sergipe. Physical Education Departament. Aracaju, SE. Brazil.

Received: January 27, 2018 Accepted: May 29, 2018 


\section{INTRODUCTION}

Mixed Martial Arts (MMA) combines techniques of different combat sports, such as: boxing, kickboxing, muay thai, wrestling, judo and Brazilian Jiu Jitsu, including motor actions in striking and grappling movements ${ }^{1}$. MMA Fights are composed of three to five rounds lasting 5' with 1 ' intervals, where winning is achieved by knockout, technical knockout, submission or judge's decision ${ }^{1-2}$. The analysis and diagnosis of technicaltactical performance in MMA can be performed through videos, providing feedback to improve performance ${ }^{2-4}$, allowing the coach to develop a higher variety of strategies which interfere in the choice and the frequency of actions taken during combat ${ }^{5-6}$.

One way to understand how combat dynamics occur and to choose the best way to prepare athletes is through time-motion analysis (TMA). TMA has been applied in many combat sports and enables describing attempts of attack, defense and decisive actions in competitive performance ${ }^{7-10}$. This information can be used in inferences about the intensity and energy demands used in combat ${ }^{7}$. In fact, when MMA athletes are consistently training at high intensity and there is no control of training load, their of injury risk increases ${ }^{1-11}$, affecting their competitive performance.

Regarding the neuromuscular component, strength and power are essential for competitive success in combat sports ${ }^{1-11-12}$. In this sense, many athletes use ergogenic substances as a way to improve performance and chances of winning, but some of those substances are considered doping ${ }^{13}$. According to Henne ${ }^{14}$, professional combat sports competitions in the United States such as Boxing and MMA partially follow the WADA (World Anti-doping Agency) code, but not all guidelines are employed. The lack of wide control over doping may encourage athletes to use illegal substances. In a study with kickboxing athletes, Sekulic et al. ${ }^{15}$ found that the behavior associated with using a doping substance increases when athletes perceive that doped fighters are not caught by the test. However, the equality of conditions and fair play should be the pillars of the sport, and doping rules are necessary for fair competition ${ }^{16}$.

The International Olympic Committee (IOC) defines doping as the administration of any exogenous substance in abnormal quantity with the intention of artificially and unfairly improving performance ${ }^{16-17}$. Although illegal substances such as anabolic androgenic steroids (AAS) increase strength and endurance, and also reduce fatigue ${ }^{16}$, the harmful effects of these substances on the health of an athlete is proven in their hormonal and aesthetic appearance as well as by their behavior ${ }^{18}$. Considering that the use of doping is associated with an improvement in physical fitness, and possibly of the motor actions involved in MMA, it is essential to investigate if there are differences in the combat actions/performances of doped athletes, whether being winners or losers. However, to the best of our knowledge no has study investigated the effect of illegal substances in MMA performances. Thus, the present study compared performances in 
three conditions through a paired technical-tactical analysis: a) positive on the anti-doping test; b) winning; and/or c) losing without doping.

\section{METHODOLOGICAL PROCEDURES}

\section{Study design}

This is a comparative and descriptive study based on retrospective data of paired athletes in MMA who had failed an anti-doping test, and analyzed the following conditions: winning without doping vs. losing without doping vs. doping (regardless of outcome). This paper specifies the technical-tactical demands of a high-performance athlete and promotes new concepts in performance measurements related to the use of illegal substances. For this, a technical-tactical analysis protocol validated and identified in previous studies $^{2-3-19}$ was adopted. Data collection was conducted from events in the Ultimate Fighting Competition (UFC ${ }^{\circledast}$ ), Pride ${ }^{\circledast}$, World Extreme Cagefight $\left(\mathrm{WEC}^{\circledast}\right)$, Elite Xtreme Combat (EliteXC $\left.{ }^{\circledast}\right)$ and Strikeforce ${ }^{\varpi}$. All episodes take place in a climatized environment except for the Ibirapuera Arena (UFC 117 ) with temperatures between $24-27^{\circ} \mathrm{C}$. The present study was submitted to and approved by the Juiz de Fora University Research Ethics Committee (protocol 51979/2017), according to the rules of resolution 466/12 of the National Health Council. According to previous studies, the study was anonymous and confidential by replacing the personal identification of athletes ${ }^{3-9-19}$.

\section{Sample}

Fifty-four (54) athletes were included in this study. The sample was composed of 267 rounds in combat (both genders). For the inclusion criterion, athletes should have failed an anti-doping test. The rounds were paired by athlete, separated by the outcome: winning $(n=90)$, losing $(n=85)$ and doping $(\mathrm{n}=92)$. The sample was obtained from professional MMA events from 2001-2014. All evaluated athletes had prior experience with the rules and procedures used during the professional events, no intervention was performed, and the combat was maintained according to the official rules.

The following procedures were adopted for the sample composition: the investigators initially searched for all reported doping cases in the professional MMA leagues. Next, all the athletes who had tested positive were identified. Combat videos were located on the internet and personal archives; when the complete video was found, two more matches (winning and losing) of the same athlete were included with the following criteria: a) the match should be in the same weight division; and b) respect the maximum time of 6 months between matches. Cases that did not meet the criteria described above were excluded. Table 1 presents the events in which athletes tested positive, date, doping agent and the outcome.

\section{Time-motion analysis protocol, intra- and inter-specialist validation}

The technical-tactical actions and the spatio-temporal changes were ob- 
Table 1. Cases in which there were athletes' failure in the anti-doping between 2001-2014, included in the present study.

\begin{tabular}{|c|c|c|c|c|}
\hline Event & Date & Doping & Rounds & Outcome of doped athlete \\
\hline UFC 30 & $23 / 02 / 2001$ & Marijuana & 1 & Winning \\
\hline UFC 36 & $22 / 03 / 2003$ & Boldenone and Nandrolone & 2 & Winning \\
\hline UFC 44 & $26 / 09 / 2003$ & Stanozolol & 1 & Winning \\
\hline UFC 48 & $19 / 06 / 2004$ & Stanozolol & 1 & Losing \\
\hline UFC Fight Night & $06 / 08 / 2005$ & Nandrolone & 3 & Winning \\
\hline UFC 62 & $26 / 08 / 2006$ & Boldenone & 3 & Losing \\
\hline Pride 32 & $21 / 10 / 2006$ & Nandrolone and Ephedrine & 2 & Winning \\
\hline Pride 32 & $21 / 10 / 2006$ & Hydroxytestosterone & 3 & Losing \\
\hline UFC Fight Night & $13 / 12 / 2006$ & Marijuana & 1 & Winning \\
\hline UFC 66 & $30 / 12 / 2006$ & Espironolactone & 2 & Winning \\
\hline WEC 35 & $20 / 01 / 2007$ & Boldenone & 1 & Losing \\
\hline Pride 33 & $24 / 02 / 2007$ & Marijuana & 2 & Winning \\
\hline UFC Fight Night & $05 / 04 / 2007$ & Cocaine & 1 & Losing \\
\hline Strikeforce & $22 / 06 / 2007$ & Boldenone and Stanozolol & 2 & Losing \\
\hline UFC 73 & 07/07/2007 & Nandrolone & 3 & Losing \\
\hline UFC 73 & 07/07/2007 & Nandrolone & 3 & Winning \\
\hline WEC 30 & 05/09/2007 & Hydrochlorothiazide & 1 & Winning \\
\hline UFC 75 & 08/09/2007 & Boldenone & 1 & Losing \\
\hline UFC 76 & $22 / 09 / 2007$ & Marijuana & 2 & Winning \\
\hline Strikeforce & $29 / 09 / 2007$ & Nandrolone, Stanozolol and Cocaine & 3 & Losing \\
\hline Strikeforce & $16 / 11 / 2007$ & Desoxymethyltestoste rone & 3 & Winning \\
\hline Strikeforce & $16 / 11 / 2007$ & Drostanolone and Nandrolone & 1 & Winning \\
\hline WEC 34 & 01/06/2008 & Boldenone & 2 & Losing \\
\hline UFC Fight Night 14 & $19 / 07 / 2008$ & Metadolone and Oxymorphine & 1 & Losing \\
\hline Elite XC & $26 / 07 / 2008$ & Boldenone & 2 & Winning \\
\hline Strikeforce & $18 / 10 / 2008$ & Drostanolone & 1 & Losing \\
\hline UFC 89 & $18 / 10 / 2008$ & Stanozolol & 3 & Losing \\
\hline UFC 94 & $31 / 01 / 2009$ & Hydrocortisone and Oxymorphine & 3 & Winning \\
\hline UFC 117 & 07/08/2010 & Testosterone & 5 & Losing \\
\hline UFC 120 & $16 / 10 / 2010$ & Stanozolol & 3 & Losing \\
\hline UFC 125 & $01 / 01 / 2011$ & Testosterone & 3 & Winning \\
\hline Strikeforce & $23 / 09 / 2011$ & Testosterone & 3 & Losing \\
\hline UFC 138 & $05 / 11 / 2011$ & Oxycodone and Oxymorphine & 2 & Winning \\
\hline Strikeforce & $17 / 12 / 2011$ & Stanazolol & 1 & Winning \\
\hline Strikeforce & 07/01/2012 & Drostanolone & 2 & Winning \\
\hline UFC 143 & $04 / 02 / 2012$ & Marijuana & 5 & Losing \\
\hline Strikeforce & $19 / 05 / 2012$ & Stanazolol & 1 & Winning \\
\hline UFC 149 & $21 / 07 / 2012$ & Ephedrin & 1 & Winning \\
\hline UFC 149 & $21 / 07 / 2012$ & Marijuana and Metadolone & 3 & Losing \\
\hline
\end{tabular}

Continue... 


\begin{tabular}{lcccc}
\hline Event & Date & Doping & Rounds & Outcome of doped athlete \\
\hline WEC 42 & $09 / 08 / 2012$ & Testoterone & 3 & Losing \\
\hline UFC 150 & $11 / 08 / 2012$ & Diuretic & 3 & Winning \\
UFC 153 & $13 / 10 / 2012$ & Drostanolone & 1 & Losing \\
\hline UFC on FUEL TV 6 & $10 / 11 / 2012$ & Marijuana & 3 & Winning \\
\hline UFC on FX 6 & $14 / 12 / 2012$ & Nandrolone & 3 & Winning \\
UFC on FUEL TV 7 & $16 / 02 / 2013$ & Marijuana & 3 & Winning \\
\hline UFC on FUEL TV 8 & $02 / 03 / 2013$ & Marijuana & 3 & Losing \\
\hline UFC 159 & $27 / 04 / 2013$ & Marijuana & 3 & Winning \\
\hline UFC 166 & $19 / 10 / 2013$ & Marijuana & 3 & Winning \\
\hline UFC Fight Night 31 & $06 / 11 / 2013$ & Marijuana & 1 & Winning \\
\hline UFC 168 & $28 / 12 / 2013$ & Human chorionic gonadotropin & 3 & Winning \\
\hline UFC on Fox 10 & $25 / 01 / 2014$ & Marijuana & 3 & Winning \\
\hline UFC Fight Night 37 & $08 / 03 / 2014$ & Marijuana & 1 & Winning \\
\hline UFC Fight Night 37 & $08 / 03 / 2014$ & Diuretic & 1 & Winning \\
\hline UFC on Fox 12 & $26 / 07 / 2014$ & Drostanolone & 1 & Winning \\
\hline
\end{tabular}

UFC - Ultimate Fighting Competition, WEC - World Extreme Cagefight, EliteXC - Elite Xtreme Combat.

served by 5 researchers in agreement with the frequency of technical and tactical actions in standing and groundwork activity, as well as the moments of high- and low-intensity following a previously established protocol ${ }^{3-9-19}$. In order to ensure ecological validity and to confirm the elite status of the athletes, the matches were analyzed using professional-quality combat records. All videos included in the analysis were of sufficient quality (standard 480/60i definition) with panoramic view of the entire competition area. When appropriate and considering the inclusion criteria, athletes were evaluated in a single competition and evaluated more than once when multiple combination videos were available, following the previously published protocol ${ }^{9-10-20}$. The reliability between the measures obtained for each technical variable was verified by the Cohen Kappa Index, and effect size (ES) and confidence interval (CI 95\%) were measured to estimate the magnitude of the differences between groups. For these analyzes, an agreement of $0.54(\mathrm{p}=0.007 ; \mathrm{ES}=0.26,95 \% \mathrm{CI}=0.5 ; 0.7)$ was observed for strikes attempted; 0.85 ( $\mathrm{p} \leq 0.001 ; \mathrm{ES}=0.26,95 \% \mathrm{CI}=0.41 ; 1.0)$ for takedown attempts; 1.0 ( $\mathrm{p} \leq 0.001, \mathrm{ES}=0.26,95 \% \mathrm{CI}=0.67,2.0)$ for chokes attempted; $1.0(\mathrm{p} \leq 0.001, \mathrm{ES}=0.26,95 \% \mathrm{CI}=0.4,2.0)$ for locks and $0.85(\mathrm{p} \leq 0.001$, $\mathrm{ES}=0.26,95 \% \mathrm{CI}=0.41 ; 1.6)$ for submission. All analyzes were processed using SPSS software (version 20.0, SPSS, Inc., Chicago, IL, USA).

\section{Data analysis}

The Kolmogorov-Smirnov test was applied to verify the normal distribution of the data. The null hypothesis was rejected $(\mathrm{p} \leq 0.05)$ for all variables. Descriptive data of the frequency of dependent variables (strikes landed, strikes attempted, takedowns landed, submissions, chokes and locks) were 
presented as mean and standard deviation (SD). For the non-parametric data, the Wilcoxon test was applied to compare results between doping vs. winning vs. losing. The effect size measurement for the non-parametric analysis was subsequently calculated, defined as ES=Z/ $\sqrt{N}$, where ES represents the effect size, $Z$ is derived from the Wilcoxon test conversion and $\mathrm{N}$ is the total number of observations. This analysis considers ES values for the effect size as small $(\mathrm{ES}<0.10)$, medium $(\mathrm{ES}<0.30)$ or large $(\mathrm{ES}>0.50)$. When appropriate, the chi-square test was used for comparisons between frequencies. Finally, an analysis of variance was applied to confirm the effects of technical-tactical actions on performance. Statistical analyses were performed using SPSS software (version 20.0) (Chicago, Illinois, USA), with a significance level of $5 \%$.

\section{RESULTS}

From the total number of matches in which an athlete failed on an anti-doping test (Table 1), 60\% ( $\mathrm{n}=34)$ ended in winning for the doped athlete $\left(X_{\text {calc }}=3.63 ; p=0.057\right)$. Seventeen (17) types of illegal substances classified as SAA $\left(55 \%, \mathrm{n}=35, \mathrm{X}_{\text {calc }}=133.509, p \leq 0.001\right)$, psychotropic $(27 \%, \mathrm{n}=17)$, thermogenic/diuretic $(9 \%, n=6)$, or opioids $(9 \%, n=6)$ were detected. Table 2 presents the paired comparisons between winning vs. losses vs. doping for effort and pause actions.

Table 2. Time motion analyze comparison between doping, winning and losing outcomes.

\begin{tabular}{|c|c|c|c|c|}
\hline \multirow[t]{2}{*}{$\begin{array}{l}\text { Time motion } \\
\text { analyze }\end{array}$} & \multirow[t]{2}{*}{ Group } & \multirow{2}{*}{$\begin{array}{c}\operatorname{mean}_{ \pm} \mathrm{SD} \\
(\mathrm{sec} .)\end{array}$} & \multicolumn{2}{|c|}{$\begin{array}{c}\text { Comparison } \\
\mathrm{P}_{\text {calculate }} ; \text { difference }(95 \% \mathrm{Cl})^{\mathrm{b}}\end{array}$} \\
\hline & & & Vs. Winning & Vs. Losing \\
\hline \multirow{3}{*}{ Groundwork } & Doping & $111.6 \pm 100.4$ & $1.0 ; 5.5(-29.4 ; 40.4)$ & $1.0 ; 10.6(-24.7 ; 46.1)$ \\
\hline & Winning & $106.1 \pm 95.4$ & & $1.0 ; 5.2(-30.1 ; 40.8)$ \\
\hline & Losing & $101.0 \pm 97.0$ & & \\
\hline \multirow{3}{*}{ Low intensity } & Doping $\$$ & $83.4 \pm 68.3$ & $0.17 ;-22.8(-51.7 ; 6.0)$ & $0.001 ;-48.3(-77.6 ;-19.1)$ \\
\hline & Winning & $106.3 \pm 77.6$ & & $0.11 ;-25.5(-54.9 ; 3.9)$ \\
\hline & Losing & $131.7 \pm 95.2$ & & \\
\hline \multirow{3}{*}{ High intensity } & Doping $\$$ & $85.2 \pm 86.6$ & $0.08 ;-26.9(-56.4 ; 2.7)$ & $0.02 ; 34.0(4.0 ; 64.0)$ \\
\hline & Winning" & $112.0 \pm 86.9$ & & $0.001 ; 60.9(30.7 ; 91.0)$ \\
\hline & Losing & $51.2 \pm 73.3$ & & \\
\hline \multirow{3}{*}{ Total time } & Doping & $253.7 \pm 87.5$ & $1.0 ;-4.8(-35.1 ; 25.5)$ & $1.0 ;-6.3(-37.1 ; 24.4)$ \\
\hline & Winning & $258.5 \pm 87.3$ & & $1.0 ;-1.5(-32.5 ; 29.4)$ \\
\hline & Losing & $260.1 \pm 79.1$ & & \\
\hline
\end{tabular}

$\mathrm{SD}=$ Standard Deviation; sec. $=$ seconds; $\mathrm{Cl}=$ Confidence Interval. Significative difference: $\#(p \leq 0.05)$ Winning vs. Losing; $\$(p \leq 0.05)$ Doping vs. Losing. bAdjusted for multiple comparison (Bonferroni).

The statistical analysis also showed a difference for the time at low-intensity $\left(F_{2,267}=11.872, p \leq 0.001, \eta^{2}=0.083\right)$, where the doping had a lower time when compared with the losing condition. For the total time at high-intensity $\left(\mathrm{F}_{2,267}=7.913, p \leq 0.001, \eta^{2}=0.057\right)$, the winning and doping presented significantly higher time versus the losing condition. Table 3 presents the comparison between the three conditions for striking actions. 
Table 3. Descriptive and statistical comparison between doping, winning and losing for striking actions.

\begin{tabular}{|c|c|c|c|c|}
\hline \multirow{2}{*}{$\begin{array}{l}\text { Time motion } \\
\text { analyze }\end{array}$} & \multirow[t]{2}{*}{ Group } & \multirow{2}{*}{$\begin{array}{c}\text { mean } \pm S D \\
(\mathrm{sec} .)\end{array}$} & \multicolumn{2}{|c|}{$\begin{array}{c}\text { Comparison } \\
\mathrm{P}_{\text {calculate }} ; \text { difference }(95 \% \mathrm{CI})^{\mathrm{b}}\end{array}$} \\
\hline & & & Vs. Winning & Vs. Losing \\
\hline \multirow{3}{*}{ Knockdown } & Doping & $0.1 \pm 0.3$ & $0.08 ;-0.09(-0.1 ; 0.2)$ & $0.6 ; 0.1(-0.19 ; 0.01)$ \\
\hline & Winning* & $0.2 \pm 0.4$ & & $0.002 ;-0.1(0.04 ; 0.2)$ \\
\hline & Losing & $0.0 \pm 0.1$ & & \\
\hline \multirow{3}{*}{$\begin{array}{l}\text { Total Strike at- } \\
\text { tempted }\end{array}$} & Doping ${ }^{\#}$ & $40.9 \pm 29.0$ & $0.01 ;-12.6(-22.9 ;-2.2)$ & $0.51 ; 6.0(-4.5 ; 16.6)$ \\
\hline & Winning* & $53.4 \pm 34.6$ & & $0.001 ; 18.6(8.0 ; 29.2)$ \\
\hline & Losing & $34.8 \pm 22.2$ & & \\
\hline \multirow{3}{*}{$\begin{array}{l}\text { Total Strike } \\
\text { landed }\end{array}$} & Doping \#\$ & $25.6 \pm 20.7$ & $0.03 ;-6.9(-13.5 ;-0.4)$ & $0.03 ; 9.3(2.6 ; 15.9)$ \\
\hline & Winning* & $32.6 \pm 20.8$ & & $0.001 ; 16.2(9.5 ; 22.9)$ \\
\hline & Losing & $16.4 \pm 11.6$ & & \\
\hline \multirow{3}{*}{$\begin{array}{l}\text { Single Strike } \\
\text { attempted }\end{array}$} & Doping ${ }^{\#}$ & $11.4 \pm 9.3$ & $0.004 ;-11.5(-20.0 ;-3.0)$ & $1.0 ;-1.4(-9.98 ; 7.3)$ \\
\hline & Winning* & $17.3 \pm 13.7$ & & $0.02 ; 10.1(1.5 ; 18.8)$ \\
\hline & Losing & $8.4 \pm 6.9$ & & \\
\hline \multirow{3}{*}{$\begin{array}{l}\text { Single strike } \\
\text { landed }\end{array}$} & Doping ${ }^{\#}$ & $24.6 \pm 19.5$ & $0.001 ;-4.2(-6.9 ;-1.6)$ & 0,$6 ; 1.4(-1.3 ; 4.1)$ \\
\hline & Winning* & $36.1 \pm 29$ & & $0.001 ; 5.6(2.9 ; 8.3)$ \\
\hline & Losing & $26.0 \pm 21.0$ & & \\
\hline \multirow{3}{*}{$\begin{array}{l}\text { Single Head } \\
\text { Strike landed }\end{array}$} & Doping $^{\#}$ & $6.6 \pm 6.3$ & $0.001 ; 4.2(-6.9 ;-1.55)$ & $0.64 ; 1.4(-1.3 ; 4.1)$ \\
\hline & Winning* & $10.9 \pm 10.1$ & & $0.001 ; 5.6(2.9 ; 8.3)$ \\
\hline & Losing & $5.3 \pm 4.9$ & & \\
\hline \multirow{3}{*}{$\begin{array}{l}\text { Single Head } \\
\text { Strike attempted }\end{array}$} & Doping ${ }^{\#}$ & $18.5 \pm 16.0$ & $0.007 ;-8.9(-15.9 ;-1.9)$ & $0.98 ;-2.8(-10.0 ; 4.2)$ \\
\hline & Winning & $27.4 \pm 23.7$ & & $0.13 ; 6.0(-1.1 ; 13.1)$ \\
\hline & Losing & $21.4 \pm 18.3$ & & \\
\hline \multirow{3}{*}{$\begin{array}{l}\text { Single Body } \\
\text { Strike landed }\end{array}$} & Doping & $2.5 \pm 3.0$ & $0.15 ; 1.0(-2.1 ; 0.2)$ & $0.18 ; 0.9(-0.3 ; 2.1)$ \\
\hline & Winning* & $3.5 \pm 4.1$ & & $0.001 ; 1.9(0.7 ; 3.1)$ \\
\hline & Losing & $1.6 \pm 2.6$ & & \\
\hline \multirow{3}{*}{$\begin{array}{l}\text { Single Body } \\
\text { Strike attempted }\end{array}$} & Doping ${ }^{\#}$ & $3.3 \pm 3.4$ & $0.04 ;-1.6(-3.1 ;-0.03)$ & $0.49 ; 0.9(-0.7 ; 2.5)$ \\
\hline & Winning* & $4.9 \pm 5.9$ & & $0.001 ; 2.5(0.9 ; 4.0)$ \\
\hline & Losing & $2.4 \pm 2.9$ & & \\
\hline \multirow{3}{*}{$\begin{array}{l}\text { Single Leg Strike } \\
\text { landed }\end{array}$} & Doping & $2.3 \pm 3.0$ & $0.4 ;-0.7(-1.8 ; 0.45)$ & $0.49 ; 0.7(-0.5 ; 1.8)$ \\
\hline & Winning* & $2.9 \pm 4.3$ & & $0.016 ; 1.4(0.2 ; 2.5)$ \\
\hline & Losing & $1.6 \pm 1.8$ & & \\
\hline \multirow{3}{*}{$\begin{array}{l}\text { Single Leg Strike } \\
\text { attempted }\end{array}$} & Doping & $2.8 \pm 3.6$ & $0.25 ;-1.0(-2.5 ; 0.39)$ & $0.9 ;-0.6(-0.8 ; 2.1)$ \\
\hline & Winning* & $3,8 \pm 5.3$ & & $0.02 ; 1.6(0.2 ; 3.1)$ \\
\hline & Losing & $2.1 \pm 2.3$ & & \\
\hline
\end{tabular}

$S D=S t a n d a r d$ Deviation; $\mathrm{Cl}=$ Confidence Interval. Significative difference: * $(p \leq 0.05)$ Winning vs. Losing; \# $(p \leq 0.05)$ Doping vs. Winning; $\$(p \leq 0.05)$ Doping vs. Losing. b Adjusted for multiple comparison (Bonferroni).

The main results of Table 3 indicate a significant effect for total Knockdowns $\left(F_{2,267}=6.007, p=0.003, \eta^{2}=0.044\right)$, where the means presented for the winning condition was higher vs. losing. A significant ef- 
fect was also observed for total attempted strikes $\left(F_{2,267}=9.331, p \leq 0.001\right.$, $\left.\eta^{2}=0.066\right)$, landed $\left(F_{2,267}=17.168, p \leq 0.001, \eta^{2}=0.115\right)$, single attempted strike $\left(\mathrm{F}_{2,267}=6.261, \mathrm{p}=0,002, \eta^{2}=0.045\right)$, landed $\left(\mathrm{F}_{2,267}=16.599, \mathrm{p} \leq 0.001\right.$, $\left.\eta^{2}=0.112\right)$, single head strike landed $\left(F_{2,267}=13.561, p \leq 0.001, \eta^{2}=0.093\right)$ and single body strike attempted $\left(F_{2,267}=7.445, p=0.001, \eta^{2}=0.053\right)$, where winning showed a higher mean in comparison to the other conditions. For single attempted head strike $\left(F_{2,267}=4.859, p=0.008, \eta^{2}=0.036\right)$, the post-hoc test indicated differences between winning and doping conditions, where winning showed a higher mean. For single body strike landed $\left(\mathrm{F}_{2,267}=7.238\right.$, $\left.\mathrm{p}=0.001, \eta^{2}=0.052\right)$, single leg strike landed $\left(\mathrm{F}_{2,267}=3.974, \mathrm{p}=0.02, \eta^{2}=0.029\right)$ and attempted $\left(F_{2,267}=3.847, p=0.023, \eta^{2}=0.028\right)$, winning showed a higher mean compared to the losing condition. Table 4 shows the comparisons for grappling actions.

Table 4. Descriptive and statistical comparison between doping, winning and losing for grappling actions.

\begin{tabular}{|c|c|c|c|c|}
\hline \multirow{2}{*}{$\begin{array}{l}\text { Time motion } \\
\text { analyze }\end{array}$} & \multirow[t]{2}{*}{ Group } & \multirow{2}{*}{$\begin{array}{c}\text { mean } \pm \text { SD } \\
(\mathrm{sec} .)\end{array}$} & \multicolumn{2}{|c|}{$\begin{array}{c}\text { Comparison } \\
\mathrm{P}_{\text {calculate; }} ; \text { difference }(95 \% \mathrm{Cl})^{\mathrm{b}}\end{array}$} \\
\hline & & & Vs. Winning & Vs. Losing \\
\hline \multirow{3}{*}{ Takedown landed } & Doping & $0.5 \pm 0.7$ & $0.6 ; 0.1(-0.4 ; 0.1)$ & $0.29 ; 0.2(-0.1 ; 0.5)$ \\
\hline & Winning* & $0.6 \pm 0.9$ & & $0.01 ; 0.3(0.1 ; 0.6)$ \\
\hline & Losing & $0.3 \pm 0.6$ & & \\
\hline \multirow{3}{*}{$\begin{array}{l}\text { Takedown at- } \\
\text { tempted }\end{array}$} & Doping & $1.1 \pm 1.5$ & $0.8 ; 0.3(-0.8 ; 0.3)$ & $1.0 ; 0.2(-0.4 ; 0.8)$ \\
\hline & Winning & $1.4 \pm 1.7$ & & $0.2 ; 0.4(-0.1 ; 1.0)$ \\
\hline & Losing & $1.0 \pm 1.4$ & & \\
\hline \multirow{3}{*}{$\begin{array}{l}\text { Submission at- } \\
\text { tempted }\end{array}$} & Doping & $0.2 \pm 0.4$ & $0.16 ;-0.2(-0.4 ; 0.04)$ & $1.0 ;-0.02(-0.2 ; 0.2)$ \\
\hline & Winning & $0.3 \pm 0.6$ & & $0.33 ; 0.1(-0.1 ; 0.3)$ \\
\hline & Losing & $0.2 \pm 0.6$ & & \\
\hline \multirow{3}{*}{ Choke attempted } & Doping & $0.1 \pm 0.4$ & $0.66 ;-0.1(-0.2 ; 0.1)$ & $0.87 ; 0.06(-0.1 ; 0,2)$ \\
\hline & Winning & $0.2 \pm 0.5$ & & $0.07 ; 0.1(-0.01 ; 0.3)$ \\
\hline & Losing & $0.1 \pm 0.3$ & & \\
\hline \multirow{3}{*}{ Lock attempted } & Doping & $0.0 \pm 0.2$ & $0.36 ;-0.1(-0.2 ; 0.1)$ & $0.43 ; 0.1(-0.2 ; 0.1)$ \\
\hline & Winning & $0.1 \pm 0.4$ & & $1.0 ; 0.01(-0.1 ; 0.2)$ \\
\hline & Losing & $0,1 \pm 0.5$ & & \\
\hline
\end{tabular}

$\mathrm{SD}=$ Standard Deviation; $\mathrm{Cl}=$ Confidence Interval. Significative difference: * $(\mathrm{p} \leq 0.05)$ Winning vs. Losing.

There was a significant effect for landed takedowns $\left(\mathrm{F}_{2,267}=4.371\right.$, $\mathrm{p}=0.014, \eta^{2}=0.032$ ), where winning showed a higher mean in comparison to the losing condition.

\section{DISCUSSION}

Anti-doping tests aim to prevent athletes from winning at any cost, thereby maintaining competitive fair play ${ }^{16}$. We do not know of any studies which have compared the performance of athletes with or without doping in 
MMA. This study conducted a primary analysis of the effect of doping on the performance of professional MMA athletes. The main results indicated that athletes who tested positive presented a higher performance in physical variables such as effort and pause time; however, the doping condition did not reflect better technical performance (Table 3 and 4), so the absence of differences in technical analysis possibly influenced the frequency of wins in doped athletes (Table 1). According to Henne ${ }^{14}$, although doping cases in other professional sports such as cycling receive increased media and academic impact, performance enhancing drugs have attracted the attention of boxing and MMA athletes.

From the total of detected cases, the majority was for AAS; in fact, the use of these substances tends to increase strength and muscle performance ${ }^{16}$. According to Hartgens et al. ${ }^{21}$, the use of AAS increases short term strength by $5-20 \%$, body mass by $2-5 \mathrm{~kg}$, and increases erythropoiesis. However, it is necessary to consider the lower number of experimental studies which measured the effect of AAS on athletic performance ${ }^{13-17}$. Baume et al. ${ }^{22}$ did not observe improvement in mental and aerobic performance after multiple doses of AAS. On the other hand, our results showed a physical advantage for doped athletes, as they presented lower pause and higher time in high-intensity actions in comparison to the losing condition. However, a lower pause time does not necessarily reflect higher chances to win, since such physical fitness advantage may represent a higher volume of motor actions applied during combat, which becomes an advantage if the combat outcome is defined by the judge's decision. However, pause can be an advantage for an athlete who wishes to finish the match (i.e. by Knockout, technical knockout or submission). According to Amtmann et al. ${ }^{7}$, actions in low-intensity effort can be used for strategic development, in which the athlete will use this period for recovery and analyze the motor actions of their opponent.

It should be noted that doping did not result in better performance for any of the measured variables when compared with winning condition. Many factors may explain lower physical performance in doping vs. winning among the technical variables. The specific literature does not show improvement in technical ability when an athlete administers any type of doping, mainly with AAS, but studies do show that the level of anger

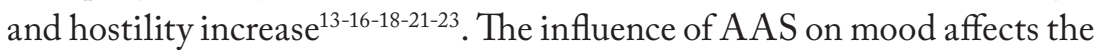
athlete"s technical ability, resulting in a lower accuracy of landed strikes (Table 3). In fact, the results of Miarka et al. ${ }^{9}$ through logistic regression reveal that effective landed head strikes are the best way to increase the chances of winning in MMA.

Another factor that limits doping in improving combat sport performance is associated with the multifactorial aspect that determines the outcome in $\mathrm{MMA}^{3-4}$. Miarka et al. ${ }^{4}$ found that winning had a higher frequency of strikes landed and submission attempts in comparison to losing athletes, especially in the third round. It is also worth noting that our analysis compared the same athletes in the three conditions; however, 
the opponent was not matched, so possibly the level of opponent in the winning condition is not the same as in losing and doping. This limitation must be considered when interpreting the results presented herein; on the other hand, it opens up a new investigative avenue for future studies. Another limitation of the present study is the heterogeneous analysis of the detected substances. There were 4 different categories, and some of which do not improve muscle power and strength such as opioids and diuretics ${ }^{25-26}$, therefore future studies should analyze each doping group separately by evaluating the effect on the performance, time and the amount used.

Finally, we emphasize that the present study is an introductory analysis on doping and MMA performance. We believe that the present work will result in future research protocols that can better elucidate the effect of these substances in a sport where many factors can determine the outcome. Our study did not show a competitive advantage and it should also be emphasized that adverse health effects can occur due to the use of doping agents such as $\mathrm{AAS}^{14-24}$. In this sense, educational campaigns can be effective, since doping may be involuntary through ingestion of contaminated supplements ${ }^{24}$. However, Sekulic et al. ${ }^{15}$ observed that kickboxing athletes with a higher level of nutritional knowledge tend to be less influenced in doping use.

\section{CONCLUSION}

In view of the aims, applied methods, results and limitations, we conclude that doping results in better physical performance when compared to a losing condition. However, doping results in lower technical performance when compared to a winning condition.

\section{REFERENCES}

1. La Bounty P, Campbell BI, Galvan E, Cooke M, Antonio J. Strength and conditioning considerations for mixed martial arts. Strength Cond J 2011;33(1):56-67.

2. Miarka B, Brito CJ, Moreira DG, Amtmann J. Differences By Ending-Rounds And Other Rounds In Time-Motion Analysis Of Mixed Martial Arts: Implications For Assessment And Training. J Strength Cond Res 2018;32(2):534-44.

3. Miarka B, Coswig V, Brito CJ, Slimani M, Amtmann J, Vecchio FBD. Comparison of combat outcomes: technical and tactical analysis of female MMA. Int J Perform Anal Sport 2016;16(2): 539-52.

4. Miarka B, Vecchio FB, Camey S, Amtmann JA. Comparisons: Technical-Tactical and Time-Motion Analysis of Mixed Martial Arts by Outcomes. J Strength Cond Res 2016;30(7):1975-84.

5. Del Vecchio FB, Hirata SM, Franchini E. A review of time-motion analysis and combat development in mixed martial arts matches at regional level tournaments. Percept Mot Skills 2011;112(2):639-48.

6. James LP, Robertson S, Haff GG, Beckman EM, Kelly VG. Identifying the performance characteristics of a winning outcome in elite mixed martial arts competition. J Sci Med Sport 2017;20(3):296-301.

7. Amtmann JA, Amtmann KA, Spath WK. Lactate and rate of perceived exertion responses of athletes training for and competing in a mixed martial arts event. J Strength Cond Res 2008;22(2):645-7. 
8. Coswig VS, Ramos Sde P, Del Vecchio FB. Time-Motion and Biological Responses in Simulated Mixed Martial Arts Sparring Matches. J Strength Cond Res 2016;30(8):2156-63.

9. Miarka B, Coswig VS, Del Vecchio FB, Brito CJ, Amtmann J. Comparisons of Time-motion Analysis of Mixed Martial Arts Rounds by Weight Divisions. Int J Perform Anal Sport 2015;15(3):1189-201.

10. Miarka B, Cury R, Julianetti R, Battazza R, Julio UF, Calmet M, et al. A comparison of time-motion and technical-tactical variables between age groups of female judo matches. J Sports Sci 2014;32(16):1529-38.

11. Tack C. Evidence-based guidelines for streng th and conditioning in mixed martial arts. Strength Cond J 2013;35(5):79-92.

12. Santana JC, Fukuda DH. Unconventional methods, techniques, and equipment for strength and conditioning in combat sports. Strength Cond J 2011;33(6):64-70.

13. Birzniece V. Doping in sport: effects, harm and misconceptions. Int Med J 2015;45(3):239-48.

14. Henne K. Defending doping: Performances and trials of an anti-doping program. J Sport Soc Issues 2016;40(3):175-96.

15. Sekulic D, Zenic N, Versic S, Maric D, Gabrilo G, Jelicic M. The prevalence and covariates of potential doping behavior in kickboxing; analysis among high-level athletes. J Hum Kinet 2017;59(1):67-77.

16. Ruiz-Rico G, Cachón J, Valdivia P, Zagalaz M. Doping vs fair play. J Sport Health Res 2014;6(1):107-16.

17. Eber N. Doping and fair play. Econ Anal Policy 2009;39(3):345-7.

18. Sjöqvist F, Garle M, Rane A. Use of doping agents, particularly anabolic steroids, in sports and society. Lancet 2008;371(9627):1872-82.

19. Miarka B, Brito CJ, Amtmann J. Performance probabilities and outcome effects in technical-tactical factors with bout phase changes of mixed martial arts. Int J Perform Anal Sport 2017;17(4):510-20.

20. Sterkowicz-Przybycien K, Miarka B, Fukuda DH. Sex and weight category differences in time-motion analysis of elite judo athletes: Implications for assessment and training. J Strength Cond Res 2017;31(3):817-25.

21. Hartgens F, Kuipers H. Effects of androgenic-anabolic steroids in athletes. Sports Med 2004;34(8):513-54.

22. Baume N, Schumacher YO, Sottas P-E, Bagutti C, Cauderay M, Mangin P, et al. Effect of multiple oral doses of androgenic anabolic steroids on endurance performance and serum indices of physical stress in healthy male subjects. Euro J Appl Physiol 2006;98(4):329-40.

23. Mottram DR. The evolution of doping and anti-doping in sport. In: Mottram DR, Chester N, editors. Drugs in sport. 7. London: Routledge; 2018. p. 43-60.

24. Rahnema C, Crosnoe L, Kim E. Designer steroids-over-the-counter supplements and their androgenic component: review of an increasing problem. Andrology 2015;3(2):150-5.

\section{CORRESPONDING AUTHOR}

Ciro José Brito

Faculdade de Educação Física e

Desporto - FAEFID

José Lourenço Kelmer st. -

Martelos, Juiz de Fora - MG, Brazil.

ZIP code: $36036-330$

E-mail: cirojbrito@gmail.com 